\title{
Therapeutic Uses of Fucoidan
}

\author{
V. Ramakrishnan ${ }^{1 *}$, S. P. Preetha ${ }^{1}$, A. Arivuchelvan ${ }^{1}$, \\ M. Parthiban ${ }^{2}$ and Thangapandiyan ${ }^{3}$
}

\author{
${ }^{1}$ Department of Veterinary Pharmacology and Toxicology, ${ }^{2}$ Department of Animal \\ Biotechnology, ${ }^{3}$ Department of Veterinary Pathology, Madras Veterinary College, \\ Chennai, India
}

*Corresponding author

Keywords

Fucoidan, Galactofucan \& Therapeutic benefits

Article Info

Accepted:

15 December 2020 Available Online: 10 January 2021
Fucoidan is a polysaccharide that primarily consists of groups of L-fucose and sulphate. As a result of its promising therapeutic results, Fucoidan is beneficial worldwide, especially in the food and pharmaceutical industries. The special biological structure is ascribed to its applaudable biological roles. Anti-oxidant, anti-tumor, anti-coagulant, antithrombotic, immunoregulatory, anti-viral, and anti-inflammatory effects are classic fucoidan-associated bioactivities. More recently, to further emphasise its therapeutic ability, a number of in vitro and in vivo experiments have been performed. This analysis reflects on the advances achieved in discovering the biological activities of fucoidan, which can be useful as a potential treatment. Therefore we have summarised the research performed in vitro and in vivo during the current decade. We expect this review and a variety of others can contribute as a theoretical basis for understanding and inspire further product development of fucoidan.

\section{Introduction}

The aquatic ecosystem is renowned as a rich source of chemical and biological diversity. This form of diversity for cosmetics, nutritional supplementation, agrochemicals and pharmaceuticals has been considered a rare source of chemical compounds (Blunt et.al., 2007). Seaweeds, such as green algae, red algae and brown algae, can produce different metabolites distinguished by a wide variety of metabolites biological processes (Rajasulochana et al., 2009). Brown algae, such as Sargassum spp., has been used as traditional Chinese medicine (TCM) for around 2000 years to treat various diseases, including thyroid diseases such as goitre. In addition, it has also been used traditionally to treat scrofula, tumours, edoema, testicular pain, swelling, cardiovascular diseases, arteriosclerosis, ulcer, renal issues, eczema, scabies, psoriasis and asthma (Liu et.al., 2012). Their therapeutic effects have been clinically approved and can therefore be explained by pharmacological actions in vivo and in vitro, such as anti-cancer, antiinflammatory, anti-bacterial, anti-viral, neuroprotective and anti-HIV development. In 
the past, numerous experiments and analyses on the bioactivity of fucoidan have been carried out, e.g. by developing anti-oxidant, anti-tumor, immunoregulation, anti-viral and anti-coagulant activities (Yoo et al., 2019).

\section{Fucoidan}

First ever extraction of fucoidan from a brown algae population was in 1913 (McDowell, 1990), such as Laminaria digitata, Ascophyllum nodosum and Fucus vesiculosus. Fucoidan is a negatively charged and highly hygroscopic polysaccharide (Cui et.al., 2018). The high content of fucoidan is found primarily in the leaves of $L$. digitata, Macrocystis pyrifera, $A$. nodosum and $F$. vesiculosus. In both water and acid solutions, Fucoidan is soluble. The number of published articles (studies) on fucoidan has increased significantly after the first publication in 1913, particularly in the modern era. The explanation for the rise in studies is that fucoidan has anti-tumor, anti-coagulant and anti-oxidant activities, as well as the importance in terms of regulating the metabolism of glucose and cholesterol (Yokota et al., 2016). Fucoidan has also been interested because of its ability to provide protection against liver damage and failure of the urinary system. As these activities are carried out, and more of its bio-activities and health-related benefits are being discovered as studies continue to accumulate, it is evident that research on fucoidan is increasingly flourishing.

\section{Sources of fucoidan}

Fucoidan is a sulfated polysaccharide present in a range of marine sources, including marine cucumbers (Mansour et al., 2019) or brown algae (Zhao et al., 2018). A great number of algae and invertebrates have been ascertained for their fucoidan contents inclusive of Fucus vesiculosus, Sargassum stenophyllum, Chorda filum, Ascophyllum nodosum, Dictyota menstrualis, Fucus evanescens, Fucus serratus, Fucus distichus, Caulerpa racemosa, Hizikia fusiforme, Padina gymnospora, Kjellmaniella crassifolia, Analipus japonicas and Laminaria hyperborea. Different types of fucoidan can be obtained from these sources and the methods of extraction used differ, especially when they are recorded in various studies.

\section{Structure of fucoidan}

Researchers have also used galactofucan in a variety of studies to represent a kind of fucoidan. The monosaccharide is known as galactofucan, and the monosaccharide structure is galactose followed by fucose, similar to rhamnofucan (rhamnose and fucose) and rhamnogalactofucan (rhamnose, galactose and fucose). There is also a difference between various forms of seaweed in addition to the structure of fucoidan. Nevertheless, fucoidan normally has two types of homofucose. One type (I) encompasses repeated $(1 \rightarrow 3)$-Lfucopyranose, and the other type (II) encompasses alternating and repeated $(1 \rightarrow 3)$ and $(1 \rightarrow 4)$-L-fucopyranose (Usoltseva et al., 2019). Reports focused on fucoidan structures sourced from various species of brown algae resulted in an enhanced structural categorisation. Most fucoidans originating from species belonging to the Fucales have an alternating of $(1 \rightarrow 3)$--L-fucose and $(1 \rightarrow 4)$-Lfucose (Lahrsen E et al., 2018). Structures of Ascophyllum nodosum fucoidan (Foley et al., 2012) and $F$. vesiculosus fucoidan show a resemblance of one another, the difference is only significant based on sulfate patterns and the presence of glucuronic acid. Similar fucoidan backbone is present in a number of Fucales species, such as Fucus serratus, Fucus distichus and Pelvetia canaliculate, but display more diversity in the branching and the presence of different monosaccharides 
(Bilan et al., 2006). There are however, exceptions, for example, fucoidans from the Bifurcaria bifucardia and Himanthalia elongate do not adopt or allocate such a structural characteristic (Jabbar Mian et al., 1973). The recognition of the fucoidan structure based on the species to which it belongs is therefore a challenge.

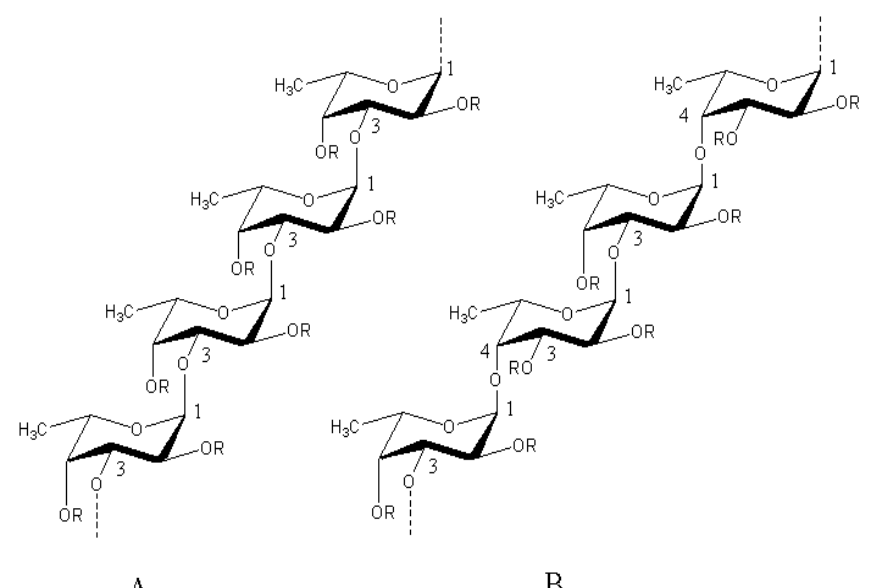

A

B

\section{Therapeutic uses}

\section{Anti - cancer capacity}

Apoptosis is a physiological mechanism known as programmed cell death and is important in organisms for embryonic growth and homeostasis, but it may also be involved in pathological processes, such as cancer. (Burz et al., 2009).This section therefore follows how malignant or cancer cells undergo apoptosis in various ways, i.e. caspases, cell cycle arrest, intrinsic and extrinsic pathways, following administration or stimulation by fucoidan. C.okamuranus Fucoidan (average Mw $75.0 \mathrm{kDa}$ ), which consists of $5.01 \mathrm{mg} / \mathrm{mL}$ of L-fucose, 2.02 $\mathrm{mg} / \mathrm{mL}$ of uronic acids and $1.65 \mathrm{ppm}$ of sulfate, has revealed that at the concentration of $1.0 \mathrm{mg} / \mathrm{mL}$, the $\mathrm{G} 0 / \mathrm{G} 1$-phase population in Huh7 hepatocarcinoma cell was increased, accompanied by a decrease in the $\mathrm{S}$ phase, highly suggesting that fucoidan may cause the cell cycle arrest at the G0/G1 phase (Nagamine et al., 2009). In a recent study by Zhang et al., a high Mw fucoidan HMWF was stated to have been isolated from Cladosiphon navae. Glysidases were then digested to obtain LMWF. A digested low molecular weight fraction $(72 \%,<500 \mathrm{kDa})$ and a nondigested fraction consisting of less than $28 \%$ were included in the LMWF $(800 \mathrm{kDa})$. Fucose $(73 \%)$, xylose $(12 \%)$ and mannose were the main constituents of LMWF (7\%).Their findings showed that the LMWF enabled apoptosis to be induced in MDAMB-231 breast cancer cells, showing a decreased cell viability trend at concentrations between 82 and $1640 \mathrm{~g} / \mathrm{mL}$, followed by further analysis of nuclear shrinkage and fragmentation, suggesting that the cytotoxic effect of the LMWF was mediated by apoptotic induction. Kasai et al., conducted comparative studies involving apoptosis, Where the type II fucoidan isolated from $F$. vesiculosus $(600 \mathrm{kDa})$ was found. In contrast to the low-molecular-weight of a type I fucoidan derivative, exhibited similar apoptosis-inducing activities via the activation of caspase -8 and -9 in MCF-7 and HeLa cells at concentrations between 10-1000 $\mu \mathrm{g} / \mathrm{mL}$. Fucoidan was also known as a probable or potential melanoma counteracting agent. While there are therapeutic methods in 
the form of a single agent or combination therapies, effectiveness depends on a variety of factors that include the patient's general health, cancer or metastasis level, and melanoma place (Domingues et al., 2018). However, due to the existence of diverse resistance mechanisms, the effectiveness of such therapies can somewhat be diminished. Therefore, new therapeutic targets for melanoma have become desperately needed. For example, $F$. vesiculosus fucoidan (purchased from Sigma) demonstrated important inhibitory effects on cell proliferation and apoptosis induction at 550 $\mu \mathrm{g} / \mathrm{mL}$ for $48 \mathrm{~h}$ on B16 melanoma cells (Wang et al., 2017). Such evidence was well executed, which was clearly evidenced by a clear contention to possess therapeutic ability on the side of fucoidan. A promising potential as a therapeutic agent is the efficacy of fucoidan to inhibit cancer cells by inducing apoptosis. It is also encouraging to note that the production of fucoidan as an anti-cancer treatment by combining it with other anticancer agents has been undertaken in a variety of clinical trials, and the limited amount of evidence collected to date seems to be leaning in a positive direction. However, serious considerations are still needed in terms of further anti-cancer research in particular the difference in outcomes between animal experiments and clinical trials in human cancer studies. This may be due to the effect of how fucoidan is consumed and processed by the body, including how fucoidan affects the body. In most cases, if not the same, these processes are identical across organisms, often they can be so different in that in one species a material may be benign but invalid to the other. Therefore it is possible to suggest some kind of multidisciplinary approach in order to achieve credible results and prevent inconsistencies between animal experiments and clinical trials as much as possible. A lot of ground still needs to be uncovered with that being said, so it is fitting to call fucoidan a 'potential therapy' rather than a cancer cure at this point before more updates are available.

\section{Therapeutic Potential against Influenza A Virus}

Between viral diseases, the flu has become one of the most problematic viruses worldwide with its seasonal outbreaks, though medicine and science are finding amicable solutions. The influenza A virus (IAV) has for example, been a formidable pathogen that has been involved in at least three pandemics since the last century. One of its featured pandemics, which was regarded to be severe, claimed at least 40 million lives worldwide 1918-1919 (Peltier et al., 1992). In late April of 2009, an influenza A virus i.e., H1N1 (Schoch-Sapna et $a l ., 2010)$, was in the limelight causing major awareness and surveillance in countries around the world. Though its prevalence was only for a brief period, its negative impact was rather great (Tognotti et al., 2013). Scientists have therefore, been searching for ways to eliminate or at least regulate IAV. Scientific analysis has expanded to investigate marine sources such as fucoidan. Recently, a Wang et al., study was conducted to inhibit Kjellmaniella crassifolia fucoidan (536 kDa, 30.1\% sulphate content) IAV infection targeting the viral neuraminidase and cellular EGFR pathway. The selection of this form of fucoidan was based on one of the other criteria that anti-IAV drug production must be highly effective and minimally or non-toxic. (Tognotti et al., 2013), hence the study on fucoidan was rather favorable as a consequence that most studies mention that fucoidan has less or no toxicity and is cost-effective compared to possible alternatives. The findings showed that K.crassifolia fucoidan blocked low toxicity in vitroIAV infection, also exhibited a wide spectrum against IAV, and displayed a low trend in viral resistance induction, outperforming the standard anti-IAV drug 
amantadine. Before infection and at some points after adsorption, K.crassifolia fucoidan was able to inactivate virus particles. This was because it could bind to viral neuraminidase (NA) as well and inhibit NA's activity to block IAV release. In IAV-infected mice, $K$. crassifolia fucoidan significantly improved survival and showed a decline in viral titers. (Sun et al., 2018) obtained two L. japonica LMWF fractions LF1 and LF2 by degradation, which contained fucose of $42.0 \%$ and $30.5 \%$; galactose content of $19.8 \%$ and $23.9 \%$; uronic acid content of $5.3 \%$ and $3.7 \%$; and sulfate content of $30.7 \%$ and $32.5 \%$, respectively. Weight-average $\mathrm{Mw}$ and numerical-average Mw were found to be 7600 and 7300 for LF1 and 3900 and 3700 for LF2, respectively. LF1 and LF2 exhibited remarkable in vitro antiviral activity especially at medium and high doses $(0.15,0.3,0.6,1.2$ and $2.4 \mathrm{mg} / \mathrm{mL})$. The in vivo findings also showed that LF1 and LF2 were capable of prolonging the survival time of virus-infected mice, and that they were capable of significantly improving the quality of immune organs, immune cell phagocytosis and humoral immunity following intravenous administration of LMWFs $(2.5,5,10$ and 15 $\mathrm{mg} / \mathrm{kg} ; 14$ days). While such fucoidans present wide-spectrum anti-viral activity, the relationship between structure and activity remains unclear. Drug resistance, toxicity and cost remain unresolved problems in the fight against IAV infection, despite the success of the currently available medications. Therefore it is of vital importance to establish novel antiIAV agents which could be used alone or in combination with existing anti-viral drugs. Such a form of fucoidan from $K$. crassifolia may be possible. Against highly pathogenic strains like H5N1 or H7N9, K. crassifoliamay be further developed. Fucoidan also has the potential to be a new nasal drop or spray for the treatment of influenza in some form and may act as prophylaxis in the near future (Moscona et al., 2009).

\section{The Role of Fucoidan as A Potential Anti-} Hepatitis B Virus Treatment

The hepatitis $\mathrm{B}$ virus is another virus that has harmful consequences (HBV). Globally, HBV infects more than 300 million people and is one of the leading causes of liver disease and cancer of the liver. The current HBV-related problem is the lack of expertise to predict the outcome and progression of HBV infection and the unmet need to understand the molecular, cellular, immunological and genetic basis of various HBV-related disease manifestations (Liang et al., 2009). A number of efforts have been made to improve the immunogenicity of $\mathrm{HBV}$ vaccines. A research was performed to investigate the HBV vaccination of Fucus evanescens fucoidan due to the fact that fucoidan was used as an adjuvant, as previously mentioned. This study found that Fucus evanescens fucoidan (130$400 \mathrm{kDa}$ ) actually acted as an adjuvant by stimulating the production of unique $\mathrm{HBV}$ surface antibodies such as HBs-AG in mice. (Kuznetsova et al., 2017).Mice were immunised with vaccine formulations containing samples of HBs-AG and fucoidan, causing serum cytokines (TNF-a, IFN-g and IL-2) to increase. Increases in the development of such cytokines have been observed in the culture of in vitro stimulated fucoidan splenocytes. The adjuvant effect of fucoidan and its derivatives was comparable to aluminium hydroxide, a standard approved adjuvant, for comparison. Based on a structural analysis, the glycosidic relation and structural characteristics of this sample are as follows: (3-a-L-Fucp (2.4-SO $\left.\mathrm{S}_{3}\right)-(1 \rightarrow 4)-\mathrm{a}-\mathrm{L}-$ Fucp $\left.\left(2-\mathrm{SO}_{3}\right)-1 \mathrm{n}\right)$. Another study showed that F. vesiculosus fucoidan was able to inhibit HBV replication both in vivo and in vitro $(\mathrm{Li}$ et al., 2017). Fucoidan suppressed the HBV replication by the activation of the EKR signal pathway and also enhanced the production of type I interferon via the activation of the host immune system. 
Another technique, which can be effectively used to prevent HBV replication, was proposed by this newly discovered mechanism. It was further noted that fucoidan can be used alone and/or synergistically to act as a new HBV treatment drug. The investigation showed that fucoidan significantly inhibited the replication of HBV in the in vivo mouse model $(100 \mathrm{mg}$ of fucoidan at $0,1,3,5$ and 7 days postinfection) and in in vitro HepG2.2.15 cells (at $<200 \mathrm{~g} / \mathrm{mL}$ ). The findings showed that $F$. Vesiculosus fucoidan can activate the MAPKERK1/2 pathway and subsequently promote IFN-alpha expression, leading to a decrease in HBV DNA and related protein production. This may indicate the possibility of using fucoidan as an alternative HBV infection therapeutic strategy. Consideration of the protection and other associated mechanisms of fucoidan, without doubt, still needs further study prior to clinical use.

\section{Therapeutic Potential as Veterinary Medicine against Canine Distemper Virus (CDV)}

Not only does viral infection limit its effects on humans, but it also affects wild and domestic animals. Although there is not a lot of evidence, there are viruses that appear to be lethal to domestic animals, such as canines (Williams et al., 2001). The downside is that there are very few and not well established treatments or medicines appropriate for such animals. This leads to certain viruses being succumbed to by such domestic pets, especially in their infancy due to a low immune system. For example, the canine distemper virus (CDV), a member of the genus morbillivirus, is a virus that infects a number of terrestrial and aquatic carnivores (Beineke et al., 2009). Presentations of respiratory and gastrointestinal disorders, accompanied by immunosuppression and neurological symptoms in infected hosts, describe this infection. (Beineke et al., 2009.A research to evaluate the anti-viral activity of fucoidan against CDV was conducted as this type of virus is very difficult to treat among canines. Fucoidan may however be part of the solution among the strategies and innovations currently under way, as the cure is not yet available. TrejoAvila et.al., 2014, reported that fucoidan extracted from $C$. okamuranus was able to inhibit CDV replication. $90.4 \%$ fucoidan was found in this extraction and its mean molecular weight was $92.1 \mathrm{kDa}$, with fucose $(38.6 \%)$, sulphate $(15.9 \%)$ and other sugars (23 percent ).Not only did it show a decrease in the number of plaques, but it also reduced their size. This fucoidan permitted a 50 percent inhibitory concentration (IC50) of 0.1 $\mu \mathrm{g} / \mathrm{mL}$ inhibition of CDV replication in Vero cells. The derived selectivity index (SI50) was $>20,000$. This showed that fucoidan is capable of inhibiting viral infection by interfering in the early stages and also by inhibiting cell fusion mediated by CDV. Fucoidan may also be useful for developing pharmacological strategies for the treatment and control of infection with CDV. Results such as these and many more to follow may be a step towards inventing canine drugs or treatments against this deadly disease.

\section{Therapeutic Potential against HIV}

One of the focal points for a number of scientists worldwide has been the search for a cure for HIV. A breakthrough in terms of the currently available medication (in the form of anti-retrovirals) for taming the virus has been noted, however. A need still remains, however to eliminate it absolutely. The current treatment challenge is related to side effects, especially during the initial introduction process. Current therapies can also be cost-prohibitive, though affected people are subsidised by some countries. In turn, in terms of exorbitant budgets, this puts 
certain limitations on governments in order to preserve people's lives. Taking this into account, research is urgently needed for novel compounds to address certain limitations. Fucoidan relies on the physical and chemical properties of its anti-viral activity. An investigation found that multiple fucoidans could suppress Jurkat cell infection using pseudo-HIV-1 particles that contain HIV-1 envelope proteins. (Profokjeva et al., 2013). Therefore, based on the data obtained, the fucoidans (Saccharina cichorioides (1.3- $\alpha-\mathrm{L}-$ fucan) and $S$. japonica (galactofucan) presented a significant inhibitory effect. This was shown by the efficacy of fucoidan at very low concentrations of $0.001-0.05 \mu \mathrm{g} / \mathrm{mL}$ against lentiviral transduction. Another antiHIV potential agent was $S$. Swartzii Fucoidan(Dinesh et al., 2016). Bioactive fucoidan fractions (CFF: Crude Fucoidan Fraction; FF1: Fucoidan Fraction 1; FF2: Fucoidan Fraction 2) were isolated from $S$. swartzii. For anti-HIV-1 properties, the fucoidan fractions were put under investigation. At concentrations of 1.56 and $6.25 \mu \mathrm{g} / \mathrm{mL}$, Fraction FF2 displayed substantial anti-HIV-1 activity, showing a $>50$ percent decrease in HIV-1 p24 antigen levels and reverse transcriptase activity. These fractions were mainly composed of sugars, sulfate and uronic acid, and the total sugar content in the FF1 and FF2 was $61.8 \%$ and $65.9 \%$; the sulfate content- $19.2 \%$ and $24.5 \%$, uronic acid- $17.6 \%$ and $13.4 \%, \mathrm{Mw}-45$ and $30 \mathrm{kDa}$, respectively. In addition, Thanh et.al., 2015, concluded that fucoidans derived from the three brown seaweeds, S. mcclurei (FSM), S. polycystum (FSP) and Turbinara ornate (FTO), also displayed similar anti-HIV activities with a mean IC50 ranging from 0.33 to $0.7 \mathrm{~g} / \mathrm{mL}$. Although the highest sulphate content was observed in FSM and their antiviral activity was not significantly different compared to the other two fucoidans, this indicates that the sulphate content of fucoidan is not an important factor for anti-HIV activities. When they were pre-incubated with the virus but not with the cells and not after infection, these fucoidans prevented HIV-1 infection, suggesting that they were able to prevent the early steps of HIV entry into target cells. Such studies are therefore an indication that fucoidans with a naturally high molecular weight, regardless of their backbone, may be effective as anti-HIV agents. While the above findings may provide a very optimistic outlook on fucoidan as an anti-HIV treatment, there is still a need for further in vitro and in vivo studies prior to clinical trials.

\section{Diabetic and Metabolic Syndrome Control}

Fucoidan has attracted some strong attention as a treatment agent for diabetes and other forms of metabolic syndrome in recent years (MetS). Extracted Fucoidan from $F$. Vesiculosus has been identified as an inhibitor of alpha glucosidase that can cure diabetes (Shan et al., 2016). Among other studies, fucoidan was mentioned to have an ability to attenuate diabetic retinopathy through inhibiting VEGF signaling (Yang et al., 2013). Additionally, a report of a low $\mathrm{Mw}$ fucoidan was noted to provide protection against diabetic associated symptoms in GotoKakizaki rats (Cui et al., 2014). Fucoidan also improves glucose tolerance by modulating AMPK signaling and GLUT4 activity (Jeong et al., 2013). Some studies mention that Fuc$\mathrm{Pg}$ (fucoidan from the sea cucumber Pearsonothuria graeffei) with an $\mathrm{Mw}$ of 310 $\mathrm{kDa}$ can be used as a form of functional food to treat MetS (Hu et al., 2014).Fuc-Pg allowed high-fat diet-fed mice to reduce weight, also reduced hyperlipidemia, and protected the liver from steatosis. At the same time, Fuc-Pg, combined with decreased macrophage penetration into adipose tissue, reduced serum inflammatory cytokines. Furthermore, it was declared that the treatment effect for MetS was primarily 
related to the 4-O-sulfated structure of fucoidan, since it was identified as a tetrasaccharide repeating unit with a backbone of $[\rightarrow 3$ Fuc $(2 \mathrm{~S}, 4 \mathrm{~S}) \alpha 1 \rightarrow 3$ Fuc $\alpha 1 \rightarrow 3$ Fuc $(4 \mathrm{~S})$ $\alpha 1 \rightarrow 3$ Fuc $\alpha 1 \rightarrow]$ n. In certain cases, with the rapid development of studies linked to intestinal microbes, fucoidan is regarded as a prebiotic to control the intestinal ecosystem or microbiome (Chen et al., 2019). It promotes the growth of beneficial bacteria which represents a mechanism inhibiting the development of MetS (Li et al., 2018). A report by Parnell et al., 2012, showed that prebiotics containing fucoidan can regulate blood glucose and metabolism by providing a beneficial environment for the growth stimulation of probiotics. Cheng et al., 2019, also demonstrated that $S$. fusiforme fucoidan (SFF) could modify gut microbiota during the alleviation of streptozotocin-induced hyperglycemia in mice. The yield of SFF was $6.02 \%$., with sulfate content up to $14.55 \%$ and the average $\mathrm{Mw}$ of $205.8 \mathrm{kDa}$. This study was done with diabetic mice where after a 6-week administration, SFF impressively decreased the fasting blood glucose, diet and water intake. In addition, the pathological changes in the heart and liver tissues were attenuated by SFF, thus enhancing liver function. In addition, in STZ-induced diabetic mice, which are manifestations associated with MetS, SFF reduced oxidative stress. At the same time, in diabetic mice, SFF significantly altered the gut microbiota, noting that SFF reduced the relative abundance of diabetesrelated intestinal bacteria, which may be the possible mechanism for relieving diabetes symptoms (Cheng et al., 2019).

Although the findings shown in this chapter seem relevant in favour of reversing diabetes and MetS, it should be noted that related research is still on going worldwide. The reasoning is based on the fact that researchers are still investigating the mechanism that enables fucoidan to reverse diabetes or MetS.
Other factors to be considered are short-chain fatty acids (SCFAs), which, after the oral intake of fucoidan, are known to play a role in providing a favourable environment in the intestine because fucoidan can not be digested by gastrointestinal enzymes, while their fermentation is considered optimal for the development of SCFAs by gut microbiota. Others may therefore research fucoidan through the disciplines of physiology or pathophysiology in the intestine, depending on interest, some may investigate the liver and/or pancreas as they play an integral role in digestion, especially in the intestine, some may examine the microbiological background, while also examining the exact mechanism of fucoidan. The truth is that the trials that are still in their early stages will have to be completed before fucoidan can be used as treatment or potential therapy for MetS and diabetes. When using a particular form of fucoidan, maybe a solid future project and/or path needs to be well defined.

\section{Anti-coagulant function}

Vascular related disease, such as ischemic heart disease, atherosclerosis and deep vein thrombosis, are still among the leading causes of death worldwide. As reported by the World Health Organization, complications associated with these diseases account for over one-quarter of death throughout the world (Raskob et al., 2014). In most cases, thrombotic related episodes are usually managed by using anti-coagulant and antithrombotic medications, such as heparin, a sulfated glycan belonging to the family of glycosaminoglycans (CAGs) (Spyropoulos et al., 2008). As it would be anticipated, such therapies tend to present with undesirable and come with severe to moderate side effects that are unavoidable (Moore et al., 2005). The side effects linked to heparin include thrombocytopenia (Baroletti et al., 2006) and hemorrhagic episodes (Clark et al., 1991), 
hence this can limit, defeat or hinder its pharmacological applications. According to evidence from available studies, it is mentioned that the anti-coagulant activity of fucoidan is dependent on its $\mathrm{Mw}$, sulfate group/total sugar ratio, sulfate position, sulfate degree, and glycoside branching (Nishino et al., 1991) and (Chandria et al., 2008) discovered that, by preparing Lessonia vadosa LMWF using free-radical depolymerization, a better anti-coagulant activity is then exhibited than the naïve fucoidan in a dose-dependent-manner. Jin et al., 2013, discovered that fucoidan's Mw and content of galactose, presented anti-coagulant activity. Documentation based on previous studies indicates that fucoidans with an Mw of $5-100 \mathrm{kDa}$ present as potential anticoagulants, while fractions greater than 850 $\mathrm{kDa}$ are lack of anti-coagulant activity (Shanmugam et al., 2000). The fucoidans with anMw ranging from $10-300 \mathrm{kDa}$, are regarded as having by far the strongest anticoagulant activity. In a previous study (Wang et al., 2010), the authors performed a comparative study of anti-thrombotic and anti-platelet activities of different fucoidans from L. japonica, where their results showed that the fucoidan of Mw 27-32 kDa exhibited a much better anti-coagulant and antithrombin activity than low molecular weight fucoidans $(3.7-7.2 \mathrm{kDa})$ through intravenous administration. A recent study (Onluchinsksya et al., 2015) reported that two dry Fucus extracts, DFE-1 and DFE-2, prepared using ultrasound technique, were investigated for their anti-coagulant activity compared to the reference agent heparin. Centered on anti-coagulant behaviours, an in vivo experiment on Wistar rats was performed to study increased blood clotting time measured by activated partial thromboplastin time (APTT) and prothrombin time (PT). The results showed that DFE-2 was similar to the anti-coagulatory effect developed by the heparin reference agent, while DFE-1 had a poor effect compared to DFE-2 or heparin. Due to their various physiochemical properties, including the content of fucoidan, the composition of monosaccharides and the variations in the contents of polyphenols and sulphate groups, the distinct anti-coagulant effect between DFE-1 and DFE-2 may be due. This was reflective of the important role of the chemical composition in the anti-coagulant activity of fucoidan.

\section{Other uses of fucoidan}

Fucoidan has a number of uses which have been mentioned below:

For people suffering from osteoarthritis, Fucoidan is very useful. Several studies in animals and humans have shown that fucoidan is beneficial for osteoarthritis. A human study found that when fucoidan extracts were administered orally, osteoarthritis was substantially reduced.

Fucoidan is beneficial for inflammation. But after inflammation, which is known as "surgical adhesions," a fibrotic reaction is triggered. These days, various newer methods have been used by specialists to treat this disease. Fucoidan is one of these processes.

Many individuals suffer from a disease known as "liver fibrosis" that is activated when toxins or contaminants penetrate the liver. Latest research has shown that fucoidan helps to reduce liver damage and prevents liver fibrosis.

Fucoidan may also be used to treat radiation exposure. We all know how harmful radiation can be. It may result in the death of cells. There are currently no human studies that indicate that fucoidan is useful for radiation protection. Several in vitro and mouse models, however, have shown that 
fucoidan is useful against radiation.

It has been seen that fucoidan plays some role in blood homeostasis. Fucoidan compounds have a retarding effect on coagulation. This is because of the interference with heparin cofactor II and antithrombin III.

Fucoidan is also useful and provides synaptic protection for the brain. In terms of safeguarding brain functioning, it has shown positive results. Various rat experiments have shown that fucoidan is necessary for the brain to function properly.

Some inhibitory effects on various viruses have also been demonstrated by Fucoidan. In 2008, a study was carried out that showed that fucoidan is helpful against viruses. (6) The analysis showed that fucoidan extracts were beneficial against infection with the herpes simplex virus.

Fucoidan has been shown to be very useful against malaria. Malaria is actually caused by a parasite called the merozoite plasmodium. In some mouse models, Fucoidan has shown good effects and has shown that it prevents plasmodium infection.

Fucoidan protects the kidneys in various ways, too. The consumption of orally administered fucoidan has been shown to be very effective in the prevention of chronic renal failure or Heymann nephritis in some studies. However the research was performed on rats.

It has also been shown that Fucoidan is useful against snake venoms. A research team from Costa Rica found that fucoidan is an important inhibitor of PLA2 variants present in the venoms of crotalid snakes.

In conclusion the fucoidan continues to promise a wide variety of future applications. Reviews and scientific papers focused on the therapeutic applications of fucoidan are on the increase since the modern period. Therefore in terms of the role Fucoidan could play as a form of therapy, support has increased. This stems from the fact that brown algae have been used to treat some TCM diseases for many years, so there is a bit of background to remember. It remains important or necessary that a specific therapeutic activity needs screening and validation for each form of fucoidan. Further evaluation also needs serious consideration of pharmacokinetics, absorption and biodistribution. There are very few clinical trials scheduled and completed, considering the numerous studies performed so far on fucoidan. This may be due to a lack of comparative studies on for example, a particular fucoidan.Studies analyse the di erent forms of fucoidan on di erent cell lines or animal models in most cases. This then makes it hard to create a general mechanism for a particular form of fucoidan. Furthermore, very little information on the absorption, distribution and excretion of fucoidan is available. The structure of fucoidans still needs attention with that being said, because they present a complex structure, even their refined structures are not yet clear or fully understood. Fucoidan's biological processes are appealing. However it is very difficult to establish the relationships between structure and operation since most of these experiments are carried out using a relatively crude fucoidan. Future studies focused on the simple conformation of fucoidan should help to enhance understanding of the biological properties of fucoidan. As a consequence of the steady increase in the availability of detection and measurement techniques, this could soon be theoretically feasible. Understanding the chemistry and structural heterogeneity of each form of fucoidan is the main element in the production of effective therapeutic products. It should be noted that some of the researchers related to in this analysis are currently carrying out further studies, 
following up on what they have previously achieved. In addition, they also suggest that future research, based on past and present work, are currently underway and will be available shortly. This paves the way for what would be considered a 'tried and tested' regimen, whether it is used as therapy one day.

\section{References}

Blunt, J.W.; Copp, B.R.; Hu,W.P.; Munro, M.H.G.; Northcote, P.T.; Prinsep, M.R. Marine natural products. Nat. Prod. Rep. 2007, 24, 31-86.

Rajasulochana, P.; Dhamotharan, R.; Krishnamoorthy, P.; Murugesan, S. Antibacterial activity of the extracts of marine red and brown algae. J. Am. Sci. 2009, 5, 20-25.

Liu, X.; Du, P.; Liu, X.; Cao, S.; Qin, L.; He, M.; He, X.; Mao, W. Anticoagulant properties of a green algal phamnantype sulfated polysaccharide and its low-molecular-weight fragments prepared by mild acid degradation. Mar. Drugs 2018, 16, 445.

Chandini, S.K.; Ganesan, P.; Bhaskar, N. In vitro antioxidant activities of three selected brown seaweeds of India. Food Chem. 2008, 107, 707-713.

Gomes, D.L.; Melo, K.R.T.; Queiroz, M.F.; Batista, L.A.N.C.; Santos, P.C.; Costa, M.S.S.P.; Almeida-Lima, J.; Camara, R.B.G.; Costa, L.S.; Rocha, H.A.O. In vitro studies reveal antiurolithic Effectof antioxidant sulfated polysaccharides from the green seaweed Caulerpa cupressoides var flabellata. Mar. Drugs 2019, 17, 326.

Liu, L.; Heinrich, M.; Myres, S.; Dworjanyn, S.A. Towards a better understanding of medicinal uses of the brown seaweed Sargassum in traditional Chinese medicine: A phytochemical and pharmacological review. J. Ethnopharmacol. 2012, 142, 591-619. Yoo, H.J.; You, D.J.; Lee, K.W.
Characterization and immunomodulatory effects of high molecular weight fucoidan fraction from the Sporophyll of Undaria pinnatifida in cyclophosphamide-induced immunosuppressed mice. Mar. Drugs. 2019, 17, 447.

McDowell, P.E. 15-Algal polysaccharides. In Methods in Plant Biochemistry, 1st ed.; Elsever: Amsterdam, The Netherlands, 1990; Volume 2, pp. 523-547.

Cui, K.; Tai, W.; Shan, X.; Hao, J.; Li, G.; Yu, G. Structural characterization and antithrombotic properties of fucoidan from Nemacystus decipiens. Int. J. Biol. Macromol. 2018, 120, 1817-1822. Yokota, T.; Nomura, K.; Nagashima, M.; Kamimura, N. Fucoidan alleviates high-fat diet-induced dyslipidemia and atherosclerosis in ApoEshl mice deficient in apolipoprotein $\mathrm{E}$ expression. J. Nutr. Biochem. 2016, 32, 46-54.

Mansour, M.B.; Balti, R.; Yacoubi, L.; Ollivier, V.; Chaubet, F.; Maaroufi, R.M. Primary structure and anticoagulant activity of fucoidan from the sea cucumber Holothuria polii. Int. J. Biol. Macromol. 2019, 121, 1145-1153.

Zhao, Y.; Zheng, Y.;Wang, J.; Ma, S.; Yu, Y.; White,W.L.; Yang, S.; Yang, F.; Lu, J. Fucoidan extracted from Undaria pinnatifida: Source for nutraceuticals/ functional foods. Mar Drugs 2018, 16, 321.

Koh, H.S.A.; Lu, J.; Zhou,W. Structure characterization and antioxidant activity of fucoidan isolated from Undaria pinnatifida grown in New Zealand. Carbohydr. Polym. 2019, 212, 178-185. Jin, W.; Wu, W.; Tang, H.; Wei, B.; Wang, H.; Sun, J.; Zhang, W.; Zhong, W. Structure analysis and anti-tumor and anti-angiogenic activities of sulfated galactofucan extracted from Sargassum thunbergii. Mar. Drugs 2019, 17, 52.

Usoltseva, R.V.; Anastyuk, S.D.; Surits, V.V.; Shevchenko, N.M.; Thinh, P.D.; 
Zadorozhny, P.A.; Ermakova, S.P. Comparison of structure and in vitro anticancer activity of native and modified fucoidans from Sargassum feldmannii and $S$. duplicatum. Int. J. Biol. Macromol. 2019, 124, 220-228.

Usoltseva, R.V.; Shevchenko, N.M.; Malyarenko, O.S.; Anastyuk, S.D.; Kasprik, A.E.; Zvyagintsev, N.V.; Ermakova, S.P. Fucoidans from brown algae Laminaria longipes and Saccharina cichorioides: Structural characteristics, anticancer and radiosensitizing activity in vitro. Carbohydr. Polym. 2019, 221, 157-165.

Lahrsen, E.; Liewert, I.; Alban, S. Gradual degradation of fucoidan from Fucus vesiculosus and its Effecton structure, antioxidant and antiproliferative activities. Carbohydr. Polym. 2018, 192, 208-216.

Zhang, Z.; Till, S.; Jiang, C.; Knappe, S.; Reutterer, S.; Scheiflinger, F.; Szabo, C.M.; Dockal, M. Structure-activity relationship of the pro- and anticoagulant effects of Fucus vesiculosus fucoidan. Thromb. Haemost. 2014, 111, 429-437.

Bilan, M.I.; Grachev, A.A.; Ustuzhanina, N.E.; Shashkov, A.S.; Nifantiev, N.E.; Usov, A.I. Structure of a fucoidan from the brown seaweed Fucus evanescens C. Ag. Carbohydr. Res. 2002, 337, 719730.

Bilan, M.I.; Grachev, A.A.; Ustuzhanina, N.E.; Shashkov, A.S.; Nifantiev, N.E.; Usov, A.I. A highly regular fraction of a fucoidan from the brown seaweed Fucus distichus L. Carbohydr. Res. 2004, 339, 511-517.

Bilan, M.I.; Grachev, A.A.; Shashkov, A.S.; Nifantiev, N.E.; Usov, A.I. Structure of a fucoidan from the brown seaweed Fucus serratus L. Carbohydr. Res. 2006, 341, 238-245.

Foley, S.A.; Szegezdi, E.; Mulloy, B.; Samali, A.; Tuohy, M.G. An unfractionated fucoidan from Ascophyllum nodosum: Extraction, characterization, and apoptotic effects in vitro. J. Nat. Prod. 2012, 75, 1674.

Descamps, V.; Colin, S.; Lahaye, M.; Jam, M.; Richard, C.; Potin, P.; Barbeyron, T.; Yvin, J.C.; Kloareg, B. Isolation and culture of a marine bacterium degrading the sulfated fucans from marine brown algae. Mar. Biotechnol. 2006, 8, 27-39.

Jabbar Mian, A.; Percival, E. Carbohydrates of the brown seaweeds Himanthalia lorea and Bifurcaria bifurcata: Part II. structural studies of the "fucans". Carbohydr. Res. 1973, 26, 147-161. Mak,W.; Hamid, N.; Liu, T.; Lu, J.; White,W.L. Fucoidan from New Zealand Undaria pinnatifida: Monthly variations and determination of antioxidant activities. Carbohydr. Polym. 2013, 95, 606-614.

Fletcher, H.R.; Biller, P.; Ross, A.B.; Adams, J.M.M. The seasonal variation of fucoidan within three species of brown macroalgae. Algal Res. 2017, 22, 7986.

Garcia-Vaquero, M.; Rajauria, G.; O’Doherty, J.V.; Sweeney, T. Polysaccharides from macroalgae: Recent advances, innovative technologies and challenges in extraction and purification. Food Res. Int. 2017, 99, 1011-1020.

Chevolot, L.; Foucault, A.; Chaubet, F.; Kervarec, N.; Sinquin, C.; Fisher, A.M.; Boisson-Vidal, C. Further data on the structure of brown seaweed fucans: Relationships with anticoagulant activity. Carbohydr. Res. 1999, 319, 154-165.

Marais, M.F.; Joseleau, J.P. A fucoidan fraction from Ascophyllum nodosum. Carbohydr. Res. 2001, 336, 155-159.

Ponce, N.M.A.; Pujol, C.A.; Damonte, E.B.; Flores, M.L.; Stortz, C.A. Fucoidans from the brown seaweed Adenocystis utricularis: Extraction methods, antiviral activity and structural studies. Carbohydr. Res. 2003, 338, 153-165.

Irhimeh, M.R.; Fitton, J.H.; Lowenthal, R.M.; Kongtawelert, P. A quantitative method to detect fucoidan in human plasma 
using a novel antibody. Methods Find. Exp. Clin. Pharmacol. 2005, 27, 705710.

Pozharitskaya, O.N.; Shikov, A.N.; Faustova, N.M.; Obluchinskaya, E.D.; Kosman, V.M.; Vuorela, H.; Makarov, V.G. Pharmacokinetic and tissue distribution of fucoidan from Fucus vesiculosus after oral administration to rats. Mar. Drugs 2018, 16, 132.

Torode, T.A.; Marcus, S.E.; Jam, M.; Tonon, T.; Blackburn, R.S.; Hervé, C.; Knox, J.P. Monoclonal antibodies directed to fucoidan preparations from brown algae. PLoS ONE 2015, 10, e0118366.

Nagamine, T.; Nakazato, K.; Tomioka, S.; Iha, M.; Nakajima, K. Intestinal absorption of fucoidan extracted from the brown seaweed, Cladosiphon okamuranus. Mar. Drugs 2015, 13, 48-64.

Tokita, Y.; Nakajima, K.; Mochida, H.; Iha, M.; Nagamine, T. Development of a fucoidan-specific antibody and measurement of fucoidan in serum and urine by sandwich ELISA. Biosci. Biotechnol. Biochem. 2010, 74, 350357.

Matsubara, K.; Xue, C.; Zhao, X.; Mori, M.; Sugawara, T.; Hirata, T. Effects of middle molecular weight fucoidans on in vitro and ex vivo angiogenesis of endothelial cells. Int. J. Mol. Med. 2005, 15， 695-699. Kadena, K.; Tomori, M.; Iha, M.; Nagamine, T. Absorption study of mozuku fucoidan in Japanese volunteers. Mar. Drugs 2018, 16, 254.

Burz, C.; Berindan-Neagoe, I.; Balacescu, O.; Irimie, A. Apoptosis in cancer: Key molecular signaling pathways and therapy targets. Acta Oncol. 2009, 48, 811-821.

Nagamine, T.; Hayakawa, K.; Kusakabe, T.; Takada, H.; Nakazato, K.; Hisanaga, E.; Iha, M. Inhibitory Effectof fucoidan on Huh7 hepatoma cells through downregulation of CXCL12. Nutr. Cancer 2009, 61, 340-347.

Zhang, Z.; Teruya, K.; Hiroshi Eto, H.;
Shirahata, S. Induction of apoptosis by low-molecular-weight fucoidan through calcium- and caspase-dependent mitochondrial pathways in MDA-MB231 breast cancer cells. Biosci. Biotechnol. Biochem. 2013, 77, 235242.

Kasai, A.; Arafuka, S.; Koshiba, N.; Takahashi, D.; Toshima, K. Systematic synthesis of low-molecular weight fucoidan derivatives and their Effecton cancer cells. Org. Biomol. Chem. 2015, 13, 10556-10568.

Domingues, B.; Lopes, J.M.; Soares, P.; Pópulo, H. Melanoma treatment in review. Immunotargets Ther. 2018, 7, 35-49.

Wang, Z.J.; Xu,W.; Liang, J.W.;Wang, C.S.; Kang, Y. Effectof fucoidan on B16 murine melanoma cell melanin formation and apoptosis. Afr. J. Tradit. Complement. Altern. Med. 2017, 14, 149-155. Peltier, M. The influenza epidemic that occurred in New Caledonia in 1921. Bull. de l'O_ce Int. d'Hygiene Publique 1922, 6, 677-685.

Schoch-Spana, M.; Bouri, N.; Rambhia, K.J.; Norwood, A. Stigma, health disparities, and the 2009 H1N1 influenza pandemic: How to protect Latino farmworkers in future health emergencies. Biosecur. Bioterror. 2010, 8, 243-254.

Tognotti, E. Lessons from the history of quarantine, from plague to influenza A. Emerg. Infect. Dis. 2013, 19, 254-259.

Wang, W.; Wu, J.; Zhang, X.; Hao, C.; Zhao, X.; Jiao, G.; Shan, X.; Tai, W.; Yu, G. Inhibition of influenza A virus infection by fucoidan targeting viral neuraminidase and cellular EGFR pathway. Sci. Rep. 2017, 7, 40760.

Sun, T.; Zhang, X.; Miao, Y.; Zhou, Y.; Shi, J.; Yan, M.; Chen, A. Studies on antiviral and immuno-regulation activity of low molecular weight fucoidan from Laminaria japonica. J. Ocean Univ. China 2018, 3, 705-711.

Moscona, A. Global transmission of oseltamivir-resistant influenza. N. Engl. 
J. Med. 2009, 360, 953-956.

Liang, T.J. Hepatitis B: The virus and disease. Hepatology 2009, 49, 13-21.

Kuznetsova, T.; Ivanushko, L.; Persiyanova, E.V.; Shutikova, A.L.; Ermakova, S.P.; Khotimchenko, M.Y.; Besednova, N.N. Evaluation of adjuvant effects of fucoidane from brown seaweed Fucus evanescens and its structural analogues for the strengthening vaccines e_ectiveness. Biomed. Khim. 2017, 63, 553-558.

Li, H.F.; Li, J.; Tang, Y.; Lin, L.; Xie, Z. Fucoidan from Fucus vesiculosus suppresses hepatitis B virus replication by enhancing extracellular signalregulated kinase activation. Virol. J. 2017, 14, 178.

Williams, E.S.; Barker, I.K. Canine distemper. Infect. Dis. Wild Mamm. 2001, 3, 5059.

Beineke, A.; $\mathrm{Pu}, \quad$ C.; Seehusen, F.; Baumgärtner,W. Pathogenesis and immunopathology of systemic and nervous canine distemper. Vet. Immunol. Immunopathol. 2009, 127, 118.

Trejo-Avila, L.M.; Morales-Martínez, M.E.; Ricque-Marie, D.; Cruz-Suarez, L.E.; Zapata-Benavides, P.; MoránSantibañez, K.; Rodríguez-Padillan, C. In-vitro anti-canine distemper virus activity of fucoidan extracted from the brown alga Cladosiphon okamuranus. Virus Dis. 2014, 25, 474-480.

Prokofjeva, M.M.; Imbs, T.I.; Shevchenko, N.M.; Spirin, P.V.; Horn, S.; Fehse, B.; Zvyagintseva, T.N.; Prassolov, V.S. Fucoidans as potential inhibitors of HIV-1. Mar. Drugs 2013, 11, 3000 3014. Dinesh, S.; Menon, T.; Hanna, L.E.; Suresh, V.; Sathuvan, M.; Manikannan, M.M. In vitro anti-HIV-1 activity of fucoidan from Sargassum swartzii. Int. J. Biol. Macromol. 2016, $82,83-88$.

Thanh, T.T.T.; Bui, M.L.; Tran, T.T.V.; Ngo, V.Q.; Ho, C.T.; Yue, Z.; Carole, S.D.; Bilan, M.U.A. Anti-HIV activity of fucoidans from three brown seaweed species. Carbohydr. Polym. 2015, 115, 122-128.

Shan, X.D.; Liu, X.; Hao, J.J.; Cai, C.; Fan, F.; Dun, Y.L.; Zhao, X.L.; Liu, X.X.; Li, C.X.; Yu, G.L. In vitro and in vivo hypoglycemic effects of brown algal fucoidans. Int. J. Biol. Macromol. 2016, 82, 249-255.

Yang,W.Z.; Yu, X.F.; Zhang, Q.B.; Lu, Q.J.; Wang, J.; Cui,W.T.; Zheng,Y.Y.;Wang, X.M.; Luo, D.L. Attenuation of streptozotocin-induced diabetic retinopathy with low molecular weight fucoidan via inhibition of vascular endothelial growth factor. Exp. Eye Res. 2013, 115, 96-105.

Cui, W.; Zheng, Y.; Zhang, Q.; Wang, J.; Wang, L.; Yang, W.; Guo, C.; Gao, W.; Wang, X.; Luo, D.L. Low-molecularweight fucoidan protects endothelial function and ameliorates basal hypertension in diabetic Goto-Kakizaki rats. Lab. Investig. 2014, 94, 382-393.

Jeong, Y.T.; Kim, Y.D.; Jung, Y.M.; Park, D.C.; Lee, D.S.; Ku, S.K.; Li, X.; Lu, Y.; Chao, G.H.; Kim, K.J. Low molecular weight fucoidan improves endoplasmic reticulum stress-reduced insulin sensitivity through AMPActivated protein kinase activation in L6 myotubes and restores lipid homeostasis in a mouse model of type 2 diabetes. Mol. Pharmacol. 2013, 84, 147-157.

Hu, S.W.; Xia, G.H.; Wang, J.F.; Wang, Y.M.; Li, Z.J.; Xue, C.H. Fucoidan from sea cucumber protects against high-fat high-sucrose diet-induced hyperglycaemia and insulin resistance in mice. J. Funct. Foods 2014, 10, 128138.

Chen, Q.; Liu, M.; Zhang, P.; Fan, S.; Huang, J.; Yu, S.; Zhang, C.; Li, H. Fucoidan and galacto-oligosaccharides ameliorate high-fat diet-induced dyslipidemia in rats by modulating the gut microbiota and bile acid metabolism. Nutrition 2019, 65, 50-59. 
Li, S.; Li, J.; Mao, G.;Wu, T.; Hu, Y.; Ye, X.; Tian, D.; Linhardt, R.J.; Chen, S. A fucoidan from sea cucumber Pearsonothuria graeei with wellrepeated structure alleviates gut microbiota dysbiosis and metabolic syndromes in HFD-fed mice. Food Funct. 2018, 9, 5371-5380.

Parnell, J.A.; Reimer, R.A. Prebiotic fiber modulation of the gut microbiota improves risk factors for obesity and the metabolic syndrome. Gut Microbes 2012, 3, 29-34.

Cheng, Y.; Luthuli, S.; Hou, L.F.; Jiang, H.J.; Chen, P.C.; Zhang, X.;Wu, M.; Tong, H.B. Sargassum fusiforme fucoidan modifies the gut microbiota during alleviation of streptozotocin-induced hyperglycemia in mice. Int. J. Biol. Macromol. 2019, 131, 1162-1170.

Raskob, G.E.; Angchaisuksiri, P.; Blanco, A.N.; Buller, H.; Gallus, A.; Hunt, B.J.; Hylek, E.M.; Kakkar, A.; Konstantinides, S.V.; McCumber, M. Thrombosis: A major contributor to global disease burden. Arterioscler. Thromb. Vasc. Biol. 2014, 34, 23632371.

Spyropoulos, A.C. Brave new world: The current and future use of novel anticoagulants. Thromb. Res. 2008, 123, 29-35.

Moore, T.J.; Cohen, M.R.; Furberg, C.D. Serious adverse drug events reported to the Food and Drug Administration, 1998-2005. Arch. Intern. Med. 2007, 167, 1752-1759.

Baroletti, S.A.; Goldhaber, S.Z. Heparininduced thrombocytopenia. Circulation 2006, 114, 355-356.
Clark, W.M.; Madden, K.P.; Lyden, P.D.; Zivin, J.A. Cerebral hemorrhagic risk of aspirin or heparin therapy with thrombolytic treatment in rabbits. Stroke 1991, 22, 872-876.

Nishino, T.; Nagumo, T.; Kiyohara, H.; Yamada, H. Structural characterization of a new anticoagulant fucan sulfate from the brown seaweed Ecklonia kurome. Carbohydr. Res. 1991, 211, 77-90.

Chandia, N.P.; Matsuhiro, B. Characterization of a fucoidan from Lessonia vadosa (Phaeophyta) and its anticoagulant and elicitor properties. Int. J. Biol. Macromol. 2008, 42, 235-240.

Jin,W.H.; Zhang, Q.B.;Wang, J.; Zhang,W.J. A comparative study of the anticoagulant activities of eleven fucoidans. Carbohydr. Polym. 2013, 91, 1-6.

Shanmugam, M.; Mody, K.H. Heparinoidactive sulphated polysaccharides from marine algae as potential blood anticoagulant agents. Curr. Sci. India 2000, 79, 1672-1683.

Wang, J.; Zhang, Q.B.; Zhang, Z.S.; Song, H.F.; Li, P.C. Potential antioxidant and anticoagulant capacity of low molecular weight fucoidan fractions extracted from Laminaria japonica. Int. J. Biol. Macromol. 2010, 46, 6-12.

Obluchinsksya, E.D.; Makarova, M.N.; Pozharitskaya, O.N.; Shikov, A.N. Effects of ultrasound treatment on the chemical composition and anticoagulant properties of dry Fucus extract. Pharm. Chem. J. 2015, 49, 183-186.

\section{How to cite this article:}

Ramakrishnan, V., S. P. Preetha, A. Arivuchelvan, M. Parthiban and Thangapandiyan. 2021. Therapeutic Uses of Fucoidan. Int.J.Curr.Microbiol.App.Sci. 10(01): 2616-2630. doi: https://doi.org/10.20546/ijcmas.2021.1001.305 